\title{
Criminologie
}

\section{Saisir la sécurité privée : quand l'État, l'industrie et la police négocient un nouveau cadre de régulation}

\section{Massimiliano Mulone et Benoît Dupont}

Volume 41, numéro 1, printemps 2008

40 ans de Criminologie : perspectives d'avenir

URI : https://id.erudit.org/iderudit/018421ar

DOI : https://doi.org/10.7202/018421ar

Aller au sommaire du numéro

\section{Éditeur(s)}

Les Presses de l'Université de Montréal

ISSN

0316-0041 (imprimé)

1492-1367 (numérique)

Découvrir la revue

Citer cet article

Mulone, M. \& Dupont, B. (2008). Saisir la sécurité privée : quand l'État, l'industrie et la police négocient un nouveau cadre de régulation. Criminologie, 41(1), 103-131. https://doi.org/10.7202/018421ar
Résumé de l'article

L'accroissement substantiel de l'industrie de la sécurité a profondément changé la manière dont la sécurité est gouvernée aujourd'hui. Une récente proposition législative de la province de Québec sur la sécurité privée - Loi sur la sécurité privée - nous a servi de point de départ pour répondre à deux objectifs, soit tenter de définir l'objet « sécurité privée » et comprendre les liens qu'entretient cette sécurité privée avec l'État et la police. Une analyse de la littérature grise accompagnant cette loi (mémoires déposés à l'Assemblée nationale du Québec et consultations particulières de la Commission des institutions) nous a permis de décrire les divers morcellements de la sécurité privée, ainsi que la difficulté à la circonscrire clairement, les frontières l'entourant étant larges et poreuses. En outre, notre analyse a mis en lumière certaines spécificités de l'État dans la gouvernance de la sécurité - soit sa capacité à légiférer et à légitimer - qui continuent à peser sur l'industrie. Enfin, il est observé que l'industrie de la sécurité privée ne tente pas tant de se substituer à la police que de se construire une place à part, qui lui soit propre et, si est possible, libre de toute contrainte.
Ce document est protégé par la loi sur le droit d'auteur. L'utilisation des services d’Érudit (y compris la reproduction) est assujettie à sa politique d'utilisation que vous pouvez consulter en ligne.

https://apropos.erudit.org/fr/usagers/politique-dutilisation/ 


\title{
Saisir la sécurité privée: quand l'État, l'industrie et la police négocient un nouveau cadre de régulation
}

\author{
Massimiliano Mulone \\ Centre international de criminologie comparée \\ Université de Montréal \\ massimiliano.mulone@umontreal.ca \\ Benoît Dupont \\ Centre international de criminologie comparée \\ Université de Montréal \\ benoit.dupont@umontreal.ca
}

RÉSUMÉ - L'accroissement substantiel de l'industrie de la sécurité a profondément changé la manière dont la sécurité est gouvernée aujourd'hui. Une récente proposition législative de la province de Québec sur la sécurité privée - Loi sur la sécurité privée - nous a servi de point de départ pour répondre à deux objectifs, soit tenter de définir l'objet «sécurité privée» et comprendre les liens qu'entretient cette sécurité privée avec l'État et la police. Une analyse de la littérature grise accompagnant cette loi (mémoires déposés à l'Assemblée nationale du Québec et consultations particulières de la Commission des institutions) nous a permis de décrire les divers morcellements de la sécurité privée, ainsi que la difficulté à la circonscrire clairement, les frontières l'entourant étant larges et poreuses. En outre, notre analyse a mis en lumière certaines spécificités de l'État dans la gouvernance de la sécurité - soit sa capacité à légiférer et à légitimer - qui continuent à peser sur l'industrie. Enfin, il est observé que l'industrie de la sécurité privée ne tente pas tant de se substituer à la police que de se construire une place à part, qui lui soit propre et, si est possible, libre de toute contrainte.

\footnotetext{
ABSTRACT - The dramatic growth of the security industry has significantly altered the way security is governed today. We relied on a recent bill on private security enacted by the province of Québec to question two dimensions, that is the definition of the object 'private security,' as seen by the state, and the existing ties of this private security with the state and the public police. Drawing from submissions (white papers)
} 
made to the parliamentary committee reviewing the Bill and from transcripts of the hearings held in Parliament, we designed a four-layer model to describe the vagueness of the delimitations of private security, as well as its inner divisions. Then, we shed some light on the capacity of the state to legislate and legitimate, thus keeping the state in a very strategic, and partially unchallengeable, position in the governance of security. Finally, we found that the private security doesn't want so much to replace the public police than create its own place in the field of security.

\section{Introduction}

Nul n'est besoin aujourd'hui de documenter la fulgurante croissance de l'industrie de la sécurité privée (Johnston, 1992; Jones et Newburn, 1998; Sanders, 2005). Son essor s'inscrit dans un contexte général de diminution du volume de l'État et de libéralisme économique (Garland, 2001) et, plus spécifiquement, dans une redirection des priorités de la police publique, d'une baisse de ses budgets et d'une augmentation de la demande en sécurité dans des sociétés où la menace terroriste, réelle ou perçue, se fait de plus en plus forte (Burbidge, 2005), le tout soutenu par des progrès technologiques qui ont dopé l'industrie de la sécurité (Brodeur, 2003).

L'implication grandissante du secteur privé dans la production et la délivrance d'un tel bien collectif - l'un des droits humains fondamentaux, le droit à la sécurité - nous oblige à nous questionner sur les possibles conséquences que ces évolutions pourraient avoir sur nos sociétés démocratiques. Parmi les auteurs qui ont cherché à comprendre et à conceptualiser cette transformation de la gouvernance de la sécurité, l'accent est souvent mis sur la place que l'État occupe (ou devrait occuper) dans le contrôle, la production et la distribution de la sécurité. L'une des propositions les plus originales et controversées à être apparues ces dernières années est celle de la gouvernance nodale telle qu'elle a été élaborée par Shearing, Johnston et leurs collègues (Shearing et Wood, 2000; Johnston et Shearing, 2003; Shearing, 2005; Wood et Shearing, 2007) ${ }^{1}$.

1. Cette conceptualisation met en avant l'émergence de collectivités privées de gouvernance de la sécurité, indépendantes des institutions étatiques, «en ce que les entités non étatiques ne sont plus simplement mobilisées par l'État, mais ont elles-mêmes développé la capacité de mobiliser et diriger d'autres ressources, incluant la police» (Shearing et Wood, $2000: 462$, notre traduction). Ces nouveaux acteurs de la production de la sécurité, ces «nœuds de gouvernance» (nodes), s'articuleraient pour former le nouveau visage de la gouvernance de la sécurité. 
Si la gouvernance nodale est avant tout une proposition normative visant à favoriser un accès démocratique à la sécurité dans des sociétés où la multilatéralisation de cette dernière menace d'augmenter des inégalités déjà existantes (Bayley et Shearing, 2001), on y trouve aussi une dimension descriptive. Pour Johnston et Shearing (2003) l'apparition de propriétés privées de masse a permis l'émergence de ce qu'ils nomment des groupes de "gouvernance corporative ${ }^{2} »$, c'est-à-dire des groupes d'intérêts privés qui produisent, distribuent et contrôlent la sécurité de manière indépendante de l'État, à l'exception de la «reconnaissance des obligations légales et constitutionnelles» (Shearing et Wood, 2000: 461, notre traduction). La position monopolistique de l'État est remise en question jusqu'à un point tel que celui-ci n'occuperait plus qu'une place équivalente aux autres acteurs de la gouvernance de la sécurité, dépassant par là même autant la vision wébérienne de l'État centralisateur que celle, néo-libérale, de l'État régulateur (Shearing, 2006). L'État ne serait plus qu'un nœud de gouvernance parmi tant d'autres et la sécurité doit être pensée de manière à dépasser la fausse nécessité qui consiste à placer les agences de régulation étatiques au centre de sa gouvernance.

Alors que d'autres auteurs défendent l'idée que l'État continue et continuera d'occuper une place particulière dans la gouvernance de ce bien collectif (Loader et Walker, 2001 ; 2006; Crawford, 2006), chacun s'entend toutefois pour dire que la simple distinction entre les secteurs privé et public n'est plus adéquate pour parler d'un champ dont la complexification des relations et la multiplication des acteurs hybrides demande un plus haut degré de conceptualisation (Johnston, 2000; Brodeur, 2003 ; Dupont, 2005 ; Crawford, 2006). Cette complexification demande à son tour d'affiner les propositions en s'appuyant sur une base empirique solide, en d'autres termes de s'affranchir temporairement de toute proposition normative pour se limiter au domaine du descriptif, autant en ce qui a trait à la sécurité privée qu'aux liens que cette dernière entretient avec certains acteurs de la sphère publique, notamment les agences de régulation étatiques et la police (Dupont, 2006a; 2006b).

Notre travail a pour principal objectif d'interroger la place de l'État dans le futur de la gouvernance de la sécurité et plus particulièrement

2. Corporate governance. 
les rapports entre sphères publique et privée, ainsi que les morcellements possibles de cette dernière. Pour répondre à ces questions, nous nous sommes penchés sur une récente proposition législative visant à encadrer le secteur de la sécurité privée. Le 14 juin 2006, le gouvernement québécois a adopté le projet de loi 88 - Loi sur la sécurité privée - (Québec, 2006a) lors d'un blitz de fin de session parlementaire. En tant qu'instrument de modernisation de la Loi sur les agences d'investigation ou de sécurité privée (Québec, 1962) elle ne tient pas seulement lieu d'aboutissement d'un long processus de transformation de l'industrie - de son volume et de son champ d'action -, mais se comprend surtout comme la pierre d'achoppement de l'avenir de la sécurité privée.

Au moyen d'une analyse de la littérature grise entourant la Loi sur la sécurité privée (consultations particulières à l'Assemblée nationale du Québec et mémoires déposés par les principaux acteurs), nous nous sommes questionnés sur le découpage des acteurs et des enjeux. Sous quelle forme l'industrie se présente-t-elle devant l'État? Quels en sont les principales alliances et les désaccords essentiels? Et, surtout, autour de quels enjeux les débats se sont-ils le plus attardés? Deux thèmes se sont ainsi dégagés de l'analyse: l'autorégulation de l'industrie (les pouvoirs alloués) et la question de la dénonciation des délits (les rapports entre les sphères publique - la police principalement - et privée, mais aussi les questions entourant le statut d'agent de sécurité au sein de la société). Ceci nous permettra de tester l'homogénéité et la cohérence interne de l'industrie de la sécurité privée, tout en mettant en question ses rapports avec la sphère publique. Tout comme nous chercherons à répondre à la question de la position qu'occupe l'État dans le champ de la sécurité.

Interroger les liens qu'entretient la sécurité privée avec l'État ne va pas sans présupposer que la première forme jusqu'à un certain point un bloc homogène et cohérent. Or, il est évident que ceci ne constitue qu'une demi-vérité, la sécurité privée étant tout aussi plurielle que peut l'être la police, si ce n'est plus (Brodeur, 2003). Dès lors, il était nécessaire de se questionner sur le morcellement possible de notre objet de travail, ses divergences internes, velléités contraires et délimitations externes. Et ce, d'autant plus que l'un des objectifs de la nouvelle loi était justement de redéfinir la sécurité privée de sorte à intégrer dans le giron de la régulation étatique toute une panoplie d'activités qui auparavant étaient exclues de toute législation. Dans ce cadre, il était intéressant de voir comment le gouvernement définissait la sécurité 
privée et comment cette dernière réagissait à cette définition, si elle acceptait cette catégorisation ou la réfutait, ce qui en bout de ligne nous informait sur la structuration de notre objet. Une seconde série de questions entourant la délimitation de l'objet et son homogénéité interne s'est donc avérée nécessaire avant de chercher à saisir les relations que la sécurité privée entretient avec la police et les agences de régulation étatique.

Notre article peut donc se comprendre comme une tentative de réponse aux deux questions suivantes:

1) Qu'est-ce que la sécurité privée? Ou, plus précisément, comment le gouvernement, au moyen de la Loi sur la sécurité privée (projet de loi 88), définit la sécurité privée et comment cette dernière réagit-elle à cette définition?

2) Quels sont les liens que cette sécurité privée entretient avec la sphère publique, notamment la police et les agences de régulation étatique (État)?

Avant d'aborder ces questions, il convient toutefois de dresser rapidement une «chronologie des événements» qui amenèrent à la Loi sur la sécurité privée, ainsi que de succinctement parcourir le contenu de cette loi. Notre approche méthodologique sera alors détaillée. Ensuite, et après avoir traité de la délimitation de notre objet, nous attaquerons les deux thèmes qui se dégagent de notre analyse, c'est-à-dire l'autorégulation de l'industrie et les rapports entre sphères publique et privée.

\section{Un long processus législatif}

La Loi sur la sécurité privée (projet de loi 88 ) a comme objectif de remplacer l'ancienne loi de 1962 sur les agences d'investigation ou de sécurité privée. Alors que celle-ci ne se chargeait que d'instaurer une obligation d'obtention d'un permis d'agent par l'entremise d'une enquête de sécurité de la Sûreté du Québec sur les antécédents criminels du candidat, la croissance extrêmement rapide du champ de l'industrie de la sécurité privée, son éclatement et sa complexification ont rendu la mise en place d'un nouveau cadre législatif nécessaire. En 1996, un comité consultatif sur la sécurité publique est mis en place. Il est composé de représentants des secteurs du gardiennage, de l'investigation, du transport de valeurs, des alarmes et des serrureries ainsi que des enquêtes de crédit (Québec, 2006b). Toutefois, ce n'est qu'à partir de 
la fin de l'année 2003 que les choses s'accélèrent avec le dépôt d'un document ministériel, le Livre blanc: La sécurité privée, partenaire de la sécurité intérieure (Québec, 2003, ci-après appelé Livre Blanc). Ce dernier, né des cendres du rapport du comité consultatif de 1996 (Québec, 2006b), crée un remous sans précédent au sein de l'industrie qui craint que le Livre Blanc, rédigé sans recours à des représentants de l'industrie, ne serve de base au prochain cadre législatif, ce document ne reflétant pas, aux yeux du secteur privé, les réalités du champ de la sécurité. Pour la première fois, on assiste à un rassemblement des forces de l'industrie, par l'entremise des principales associations professionnelles, pour chercher à modeler la future législation. Cela débouche, en juin 2004, sur la création, par le ministre de la Sécurité publique, d'un comité directeur et de cinq comités sectoriels - investigation, sécurité interne, gardiennage, alarme et serrurerie et transport de valeurs - auxquels la sécurité privée est conviée à participer, mais point la police, qui pourtant avait elle aussi émis des critiques à la suite du Livre Blanc. La remise des rapports des comités sectoriels en septembre $2004^{3}$ servira de base au dépôt du projet de loi 88 - Loi sur la sécurité privée, le 16 décembre 2004.

L'année 2005 sera marquée par quatre consultations particulières à la Commission des institutions de l'Assemblée nationale sur le projet de loi $88^{4}$. Le 18 octobre, le rapport final de consultation est déposé. Enfin, après quelques modifications, la loi est adoptée le 14 juin 2006, durant une fin de session parlementaire caractérisée par le vote de plusieurs lois controversées 5 .

3. Ces rapports sont encore disponibles sur le site du ministère de la Sécurité publique au www.msp.gouv.qc.ca/police/publicat/comites_sectoriels/ (consulté le 12 septembre 2007).

4. Ces consultations eurent lieu les 17 février, 17 mars, 13 et 15 septembre 2005.

5. Pas moins de quatre autres lois furent soumises au vote de l'Assemblée ce jour-là, dont deux (le projet de loi 125 - Loi modifiant la Loi sur la Protection de la jeunesse et d'autres dispositions législatives - et le projet de loi 1 - Loi sur la réduction de la dette et instituant le Fonds des générations) virent leur vote reporté au lendemain du fait de la virulence des débats et d'une demande de vote par appel nominal (pour plus de détails se référer au site de l'Assemblée nationale http://www.assnat.qc.ca/fra/37legislature2/Debats/journal/ $\mathrm{ch} / 060614 . \mathrm{htm})$. Ajouté à cela une fin de session parlementaire prévue pour le lendemain, la loi 88 fut adoptée dans une relative indifférence des parlementaires et des médias. 


\section{La Loi sur la sécurité privé}

Le projet de loi 88 - Loi sur la sécurité privée, tel qu'il a été déposé en décembre 2004, est composé de 136 articles découpés en 9 chapitres: (I) Champ d'application et interprétation; (II) Permis; (III) Bureau de la sécurité privée; (IV) Dénonciation et rapport; (V) Pouvoirs du ministre; (VI) Pouvoirs réglementaires; (VII) Dispositions pénales; (VIII) Dispositions de concordance et transitoire; (IX) Dispositions finales. La version finale adoptée au mois de juin 2006 (Québec, 2006a) ne comportera plus que 134 articles et un chapitre entier sera amputé (le chapitre IV portant sur la question des dénonciations). Nous reviendrons régulièrement en cours d'article sur les modifications qui ont fait suite aux consultations particulières sur la loi.

Son principal objet est la création d'un Bureau de la sécurité privée aux pouvoirs réglementaires étendus. Celui-ci doit être composé de 11 membres, dont sept sont «nommés par les associations représentatives de la sécurité privée reconnues par le ministre» (Québec, 2006a, art. 44.2) et les quatre autres par le ministre de la Sécurité publique. Sa mission générale est «de veiller à la protection du public» (Ibid, art. 40) et, à cette fin, il s'occupe de délivrer les permis d'agent et d'agence, de traiter des plaintes et de veiller au respect des dispositions de la présente loi. Il possède en outre des pouvoirs d'inspection et d'enquête importants pour atteindre ces objectifs (Ibid, art. 68-74). Un inspecteur du Bureau peut par exemple «examiner et tirer copie des livres, registres, comptes, dossiers et autres documents se rapportant aux activités du titulaire de permis» (Ibid, art. 69.2). En outre, «[c]e projet de loi confie divers pouvoirs réglementaires au Bureau, dont celui de déterminer les normes de comportement applicables aux titulaires de permis d'agent, et au gouvernement» (Ibid, notes explicatives). Autofinancé (à même les droits versés par les titulaires de permis), le Bureau reste sous le pouvoir du ministre, en ce que ce dernier peut à tout moment déléguer quelqu'un pour remplacer le conseil d'administration. Il n'en reste pas moins que ceux qui y siègeront posséderont une position cruciale au sein de l'industrie de la sécurité privée. C'est d'ailleurs autour du Bureau, de sa constitution et de ses pouvoirs, que les discussions seront les plus nombreuses.

En sus du pouvoir de destituer le conseil d'administration du Bureau, le ministre est en charge des décisions concernant l'identification extérieure (uniformes et véhicules de la sécurité privée) et la formation 
requise par les agents ${ }^{6}$. La question de l'uniformisation des exigences requises pour devenir agent de sécurité est un second élément d'importance du projet de loi. En effet, jusqu'à présent, une simple enquête de sécurité de la Sûreté du Québec suffisait à la délivrance d'un permis d'agent ${ }^{7}$. Or, le gouvernement veut établir des normes précises, étendues à l'ensemble de la province, pour mieux contrôler la qualité de titulaires de permis d'agent ainsi que les actionnaires des entreprises de sécurité. Toutefois, le projet de loi se limite à faire état d'une telle volonté, sans préciser plus loin la nature des exigences des futures formations, celleci devant encore être décidée aujourd'hui.

La loi se donne aussi comme objectif de définir les activités qui relèvent de la sécurité privée et qui doivent être incorporées ou exclues de la loi (Ibid, chapitres I et II). En identifiant les entreprises qui doivent se munir d'un permis d'agence et les individus qui doivent obtenir un permis d'agent, elle délimite un champ dont les frontières sont traditionnellement floues et propose sa propre vision de ce qui est de la sécurité privée et ce qui n'en est pas.

Plus qu'une loi, c'est un cadre de régulation que propose le gouvernement. L'objectif annoncé est l'autorégulation de l'industrie pour, d'une part, éviter des coûts supplémentaires et, d'autre part, poser les bases de son épanouissement. L'idée d'un partenariat public-privé, idée soulevée lors de la rédaction du Livre Blanc - sous-titré La sécurité privée, partenaire de la sécurité intérieure - est d'abord limitée au strict minimum, pour enfin être totalement évacuée de la version finale de la loi, alors que l'on donne au Bureau, majoritairement dirigé par l'industrie, des pouvoirs et des responsabilités suffisantes pour permettre son autonomie.

\section{Méthodologie}

Dès le dépôt du projet de loi 88, en décembre 2004, les acteurs impliqués dans le domaine de la sécurité privée furent invités à participer à des consultations particulières de la Commission des institutions de l'Assemblée nationale du Québec, ainsi qu'à envoyer des mémoires à la même commission en vue d'essayer d'améliorer la loi et d'éviter de trop

6. Sur ce dernier point toutefois les décisions se prendront en consultation avec le Bureau et l'industrie.

7. Du moins pour le gardiennage. En ce qui concerne le transport de valeurs, la question est plus complexe. 
grands mécontentements. C'est sur ces deux sources documentaires que nous nous sommes appuyés pour analyser les enjeux actuels de l'industrie de la sécurité privée.

Les 35 mémoires déposés à l'Assemblée nationale, longs de 22 pages en moyenne, peuvent être grossièrement séparés en trois catégories. Tout d'abord, et en majorité, se trouvent les acteurs appartenant à l'industrie de la sécurité privée ${ }^{8}$, c'est-à-dire les représentants d'associations professionnelles (CANASA, ASIS, AQIS, etc.; le détail des participants et des abréviations se trouve en annexe), d'entreprises (Garda, Brink's), d'une institution scolaire, de deux particuliers et des regroupements syndicaux.

Ensuite vient ce que nous pouvons nommer l'industrie parallèle' constituée de représentants d'entreprises dont l'objet principal n'est pas la sécurité, mais qui pourraient être touchées par la Loi sur la sécurité privée. Ce sont les associations de banques $(\mathrm{ABC})$, d'assurances (Bureau d'assurance du Canada) et d'agent immobiliers (ACAIQ), ainsi que certaines associations d'intérêts variés tels que le Conseil du patronat du Québec, le Conseil québécois du commerce de détail (CQCD), le Centre de recherche-action sur les relations raciales (CRARR) ou encore la Confédérations des syndicats nationaux (CSN). L'une des requêtes principales de ces acteurs sera, comme nous le verrons par la suite, d'être exclus de l'application de la Loi sur la sécurité privée.

Enfin, l'on trouve en minorité la police, représentée par différents regroupements syndicaux ${ }^{10}$. Alors qu'il faut immédiatement noter l'absence de représentants des chefs de police, les syndicats, eux, s'impliquèrent plus sérieusement dans le processus législatif.

Notre analyse s'est toutefois attardée plus en profondeur sur les retranscriptions des quatre consultations particulières qui eurent lieu à la Commission des institutions de l'Assemblée nationale du Québec les 17 février, 17 mars, 13 et 15 septembre 2005. Ces consultations totalisèrent approximativement 18 heures pour 23 participants, constitués en majeure partie de membres de l'industrie de la sécurité privée et de syndicats policiers qui avaient auparavant déposé un mémoire. Découpés en tranches de 45 minutes ( 15 minutes de présentation des principales requêtes, 15 minutes de questions des représentants du gouvernement,

8. 17 mémoires, 387 p., méd $=22,7$ [4-65]; $=16,87$.

9. 14 mémoires, 290 p., méd $=20,7[1-140] ; \_=35,71$.

10. 4 mémoires, 94 p., méd $=23,5[16-30] ; \_=7,55$. 
15 minutes de questions des représentants de l'opposition officielle), ces débats présentaient l'avantage de se trouver en bout de ligne du processus législatif et de condenser l'information en sus. Le temps limité alloué aux différents intervenants, tout comme le principe de discussions qui s'ensuivait, permettaient de mieux saisir l'essence des enjeux principaux.

À partir de l'ensemble de ces documents, disponibles sur le site de l'Assemblée nationale du Québec ${ }^{11}$, nous avons procédé à une analyse documentaire au moyen du logiciel d'analyse qualitative NVivo en cherchant à dégager les principaux thèmes abordés pendant les débats ${ }^{12}$. C'est aussi à partir de ces données que l'on a pu analyser le processus de délimitation de l'objet «sécurité privée», dont nous allons discuter maintenant.

\section{Problèmes de définitions}

Jusqu'à présent, nous avons parlé de l'industrie privée comme d'un tout, le plus souvent confronté à la police et à l'État, eux aussi conceptualisés en un groupe homogène et fermé. Il est aisé de taxer cette conceptualisation d'abusive étant donné qu'il y a peu de chances que la multitude d'acteurs qui constituent la sécurité privée agissent dans une même logique, selon des intérêts communs et avec les mêmes objectifs. En outre, il s'agit de décider où commencent et où s'arrêtent les activités dites de sécurité privée. En d'autres termes, parler de relations entre la sécurité privée et l'État ou entre la sécurité privée et la police, présuppose que chacun de ces acteurs possèdent des délimitations suffisamment claires et des divergences internes - notamment dans les buts poursuivis - suffisamment faibles pour constituer un tout cohérent. Or, s'il est nettement plus connu que l'État et la police sont des entités

11. www.assnat.qc.ca. L'adresse exacte à laquelle nos documents peuvent encore être consultés est: http://www.assnat.qc.ca/Indexweb/Recherche.aspx?cat $=s v \&$ Session $=j \mathrm{jd} 3$ 711 se $\&$ Section $=$ projlois $\&$ Requete $=88 \% 20$ - $\% 20$ Loi $\% 20$ sur $\% 201$ a $\% 20$ sécurité $\% 20$ privé.

12. Sans partir avec une consigne précise, nous avons opté pour une démarche exploratoire, par synthèse thématique, des préoccupations et requêtes abordées devant la Commission des institutions. Naturellement, certaines d'entre elles s'avérèrent partagées par un grand nombre de participants, alors que d'autres ne relevaient que d'une seule intervention. Ce sont les premières qui retinrent le plus notre attention et qui contribuèrent majoritairement à la constitution du présent article. Il est à noter que les trois sujets principaux de cet article (la constitution du Bureau, les relations entre secteurs public et privé et la définition de la sécurité privée) furent abordés par la presque totalité des participants. 
plurielles et éclatées (Brodeur, 2003; Jones et Newburn, 2006), cela est moins documenté pour l'industrie de la sécurité privée.

Il nous semble ainsi pertinent d'essayer de décrire comment la loi délimitait l'objet «sécurité privée» et comment ce dernier a réagi à ce processus d'inclusion-exclusion, de quelle manière les interactions entre les membres du gouvernement et les représentants de l'industrie ont défini la sécurité privée au Québec. Ceci nous a amenés à saisir les frontières qui délimitent la sécurité privée comme une suite de couches plus ou moins perméables et mouvantes, tel qu'il a été illustré à la figure 1.

\section{F I G U RE 1}

\section{Délimitations et divisions de la sécurité privée} selon le projet de loi 88

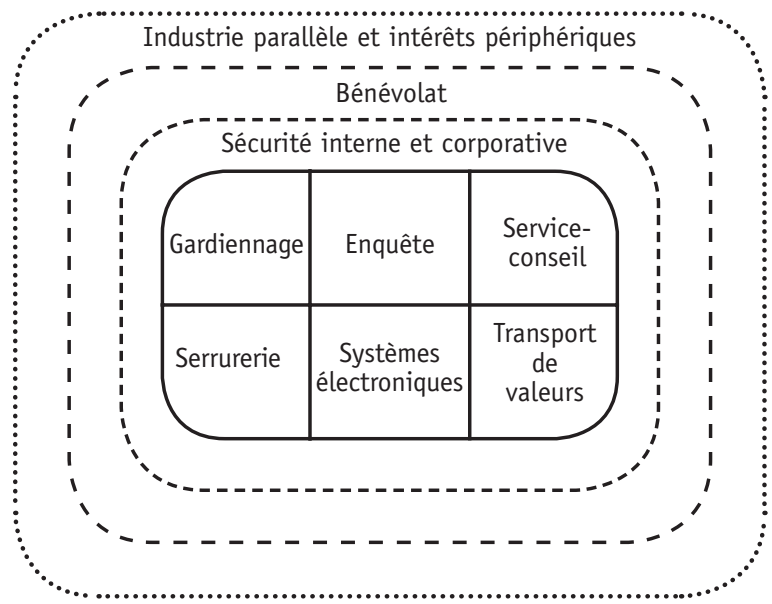

De manière grossière, le projet de loi définissait la sécurité privée par une brève énumération de six différents types d'activité: le gardiennage, l'investigation, l'industrie de la serrurerie, celle des systèmes de surveillance électronique, le convoyage de biens de valeurs et le service de conseil en sécurité ${ }^{13}$. Or, certaines entreprises cherchèrent explicitement à se dérober de la loi, car celle-ci «prévoit notamment qu'une personne

13. Dans la version finale, la sectorisation sera réduite à cinq types d'activités, les industries de la serrurerie et des systèmes de surveillance électronique se trouvant fusionnées (ce qui apparaît sur la figure 1 en pointillé). Cela est dû au fait que trop d'entreprises pratiquent les deux activités de manière peu différenciée et que les coûts d'un double permis d'agence ne se justifient dès lors que difficilement. 
qui exploite une entreprise offrant une des activités visées doit être titulaire d'un permis d'agence. La personne physique qui exerce une activité de sécurité privée ainsi que son supérieur immédiat doivent être titulaires d'un permis d'agent» (Québec, 2006a, chapitre II.1, art. 16). Cette proposition, synonyme de coûts supplémentaires ${ }^{14}$, a fortement inquiété plusieurs acteurs qui se trouvaient de manière périphérique dans le champ de la sécurité, telle que certaines entreprises d'électronique dont la pose d'alarmes (une activité de sécurité) ne constitue qu'une frange marginale de leurs activités commerciales. Dès lors, l'exception suivante a été ajoutée à la version finale de la loi : «[c]ependant, si cette personne exerce une telle activité pour le compte exclusif d'un employeur dont l'entreprise ne consiste pas à offrir une activité de sécurité privée, elle n'est tenue d'être titulaire d'un permis d'agent que s'il s'agit de son activité principale» (Ibid, chapitre II.1, art. 16).

L'exclusion des services internes de sécurité ${ }^{15}$, notamment via les représentants d'institution financière, d'assurances et de propriétaires immobiliers, fut aussi matière à débat. Du côté du secteur privé, on arguait que leurs activités de sécurité se déroulant «à l'interne», c'està-dire sans contact avec le public, il n'était pas nécessaire de les inclure dans le projet de loi. Cet argument ne fut pas retenu par les législateurs, même si le permis d'agence n'a pas été considéré comme obligatoire pour les services à l'interne, l'inclusion dans la nouvelle loi ne se faisant qu'au travers de l'obligation d'obtention d'un permis d'agent dont l'activité principale en est une de sécurité. Il reste à voir, à la suite des

14. Non seulement il faut payer pour le permis d'agence et pour chaque permis d'agent, mais ceci s'accompagne d'exigences de formation elles-mêmes coûteuses. En outre, la réputation de l'entreprise doit être parfaite, dans le sens où chaque personne exerçant une activité de sécurité, ainsi que l'ensemble des dirigeants de l'entreprise (et même des actionnaires principaux) se doivent de ne pas avoir été reconnus coupables d'une infraction criminelle (à moins d'avoir obtenu un pardon). Or, si cela n'est pas nouveau pour les entreprises de sécurité privée qui étaient soumises à la loi de 1962, cela peut poser des problèmes importants pour certaines organisations nouvellement incluses, ne serait-ce qu'en termes de gestion de personnel.

15. Un service interne de sécurité correspond à la division en charge de la sécurité au sein d'une entreprise dont l'activité principale n'est pas liée à la sécurité. Les banques ou les gestionnaires d'immeubles en sont des exemples. En opposition aux services contractuels - des entreprises qui vendent des services ou du matériel de sécurité à des particuliers -, le service interne dépend du budget alloué par l'entreprise à la protection des personnes, du matériel et de l'information. Il arrive souvent que ces services sous-traitent une partie de la sécurité (notamment au regard du personnel de gardiennage et des technologies de surveillance), mais il est plus rare qu'ils vendent leurs services à l'externe. 
inquiétudes de la CSN, si cette notion d'«activité principale» sera facilement contournée par les entreprises.

Troisième argument d'exemption, celui de la compétence fédérale, a lui aussi été déployé par les institution bancaires et d'assurances. Pour les entreprises de taille nationale (et internationale), le fait de devoir se plier à tout un ensemble de différentes exigences provinciales, en matière de formation notamment, représente des complications financières et de gestion importantes. D'autant plus que certaines de leurs activités se déroulent nécessairement sur un plan trans-provincial, notamment en ce qui concerne le domaine des enquêtes ou du transport des valeurs. L'arrêt Bell Canada c. Québec (Québec, 1989) a d'ailleurs statué sur la compétence d'une province à légiférer au sujet des entreprises interprovinciales et a été brandi comme argument d'exemption par Brink's, l'un des plus gros acteurs dans le domaine du transport de valeurs au Canada. Selon cette entreprise, «une loi provinciale ne peut [...] avoir pour effet de toucher à une partie essentielle de la gestion et de l'exploitation d'une entreprise interprovinciale», et elle «entend [...] démontrer que plusieurs articles du Projet de loi $n^{\circ} 88$ réglementent inévitablement des parties essentielles de la gestion et de l'exploitation de son entreprise» (Brink's Canada ltée, 2005: 4-5).

Paradoxalement, Brink's demanda une représentation au Bureau de la sécurité, alors même qu'elle espérait pouvoir se faire exempter de la loi que ce même Bureau serait en charge de faire respecter.

La défense de l'exemption par compétence fédérale n'a été que très partiellement entendue, avec une légère modification de formulation concernant les enquêtes entreprises à l'extérieur de la province et qui s'étendent au territoire du Québec. Ainsi, à la première distinctioninclusion traditionnelle - par type d'activités - s'ajoute une seconde couche basée sur la distinction entre services internes de sécurité et services contractuels. En outre, le fait d'opérer à un niveau national ajoute une complexité supplémentaire due à la nécessité de composer avec une multitude d'exigences différentes. Il est à noter que cela n'est pas seulement en rapport avec la taille de l'entreprise en question, mais aussi à la nature de ses activités (le transport de valeurs par exemple).

Les frontières de la sécurité privée s'élargissent encore plus lorsqu'on tient compte de la question du bénévolat, question largement abordée par de nombreux acteurs de l'industrie. Nombre d'activités de sécurité sont assurées par des personnes bénévoles. On pense notamment aux divers individus en charge de la sécurité et portant une identification 
extérieure officielle lors de manifestations sportives, de rassemblements culturels et autres. Le bénévolat a été explicitement exclut de la Loi sur la sécurité privée (Québec, 2006a, art. 2.8) et cela a fait réagir plusieurs représentants de l'industrie qui voulaient contraindre les volontaires aux mêmes exigences que les professionnels et ce dans un souci de protection du public. Bien qu'il soit plus probable que des intérêts d'ordre pécuniaire soient à l'origine de cette requête ${ }^{16}$, la présence importante du volontariat dans la provision de la sécurité implique un troisième niveau d'activités dans le champ de la sécurité, activités de plus en plus en marge il est vrai, mais qui en restent partie prenante.

Enfin, aux abords de la sécurité privée se dessinent tout un ensemble d'intérêts périphériques, constitués en bonne partie par ce que nous avons nommé «l'industrie parallèle». Sans forcément produire de la sécurité, de nombreux acteurs sont plus ou moins en situation d'être touchés par la nouvelle législation, ayant quelques intérêts dans le domaine. Ainsi, des regroupements comme le Conseil du patronat du Québec, la Chambre de la sécurité financière, la Fédération canadienne de l'entreprise indépendante ou encore la CSN ont fait entendre leur voix sur des préoccupations le plus souvent circonscrites à des objets très précis. Sans être partie prenante de la sécurité privée, chacun de ces acteurs est suffisamment en rapport avec le milieu pour être en situation de subir un impact, même mineur, de la nouvelle législation.

La difficulté d'atteindre une définition consensuelle de la sécurité privée ne se limite donc pas aux exercices didactiques, mais se prolonge largement dans les agences de régulation étatiques. Là aussi, on procède par "énumération», on se contente de dresser une liste de différentes activités que l'on catégorise comme étant de la sécurité privée, sans véritablement s'attarder à l'essence de l'objet. Rien ne nous est dit ainsi sur ce qu'est de la sécurité ou ce qui distingue le privé du public, difficultés pourtant bien réelles et problématiques, considérant l'absence de frontières claires et précises. D'autant plus que les définitions de chacune des activités énumérées peuvent elles aussi être matière à débat.

Les quatre couches qui englobent l'objet «sécurité privée» ne constituent qu'une première fragmentation grossière de l'industrie. Elle

16. Ainsi, si le bénévolat devait être inclus dans la loi, ce serait probablement sa fin (qui voudrait payer une formation et un permis d'agent pour ensuite exercer cette activité sans rémunération?), ce qui obligerait ceux qui y ont traditionnellement recours de se tourner vers les entreprises privées pourvoyeuses de services de sécurité, signifiant par la même une augmentation du chiffre d'affaire. 
devrait s'accompagner d'un travail de comparaison avec d'autres législations et avec le terrain, ainsi que d'une réflexion sur la nature de la sécurité. Notre exercice se révèle toutefois utile pour mettre en lumière plusieurs éléments. Tout d'abord, elle nous permet de saisir à quel point la marge de négociation qui existe dans toute législation sur la sécurité privée est grande. Il y a tant de différentes activités qui peuvent être considérées comme devant être régulées et tant de résistances de nature différente que le travail de circonscription de l'objet sur lequel il faut légiférer est toujours difficile. D'autant plus que même les activités centrales (les cinq secteurs), celles qui devraient pourtant être le moins matière à débat sont sources d'incertitudes et d'imprécisions (comme nous le montre, par exemple, le débat sur la définition du transport de valeurs). En outre, on ne peut que s'interroger sur l'absence de certains secteurs - les entreprises d'enquêtes de crédit et de renseignements privés en tête - qui semblent pourtant relever sans conteste d'une activité de sécurité privée (Commission du droit du Canada, 2006).

Ensuite, cela permet de saisir la multiplicité de la sécurité privée et les intérêts divergents, voire contradictoires, qui l'entourent. Toutefois, si cela nous oblige à être plus prudents lorsque l'on parle de relation entre la sécurité privée et la police ou l'État, il n'en reste pas moins, comme nous allons le voir maintenant, que sur certains sujets, l'ensemble des intervenants ont réagi d'une manière identique, notamment sur la question de l'obligation de dénonciation, donnant un semblant d'identité commune à ce que nous nommons l'industrie de la sécurité privée.

\section{Un débat houleux}

Si la sécurité privée est un objet pluriel et que, en conséquence, ses relations avec la sphère publique sont elles aussi multiples, il n'en reste pas moins que certains sujets abordés au cours des diverses consultations de la Commission des institutions de l'Assemblée nationale du Québec donnèrent lieu à des réactions très homogènes de la part de l'ensemble des participants. Parmi ceux-ci, deux sujets nous ont plus spécifiquement aidés à saisir les relations existantes entre la sécurité privée et les agences gouvernementales, notamment la police et le ministère de la Sécurité publique: l'autorégulation de l'industrie et la tentative, avortée, d'instauration d'une obligation de dénonciation. 


\section{Autorégulation et constitution du Bureau}

L'objectif premier de la Loi sur la sécurité privée, du moins aux yeux du gouvernement, est de permettre et de favoriser l'autorégulation de l'industrie. Comme le souligne le ministre de la Sécurité publique de l'époque, M. Jacques Chagnon, en s'adressant à l'industrie:

L'idée globale du projet de loi, je pense que vous l'avez comprise, [...] c'est un processus d'autorégulation dans lequel évidemment vous allez avoir suffisamment d'espace et de capacité pour pouvoir faire ce genre de démarche là. (Consultation 1, Chagnon)

De cette manière, on vise la mise en place d'une structure, dont le noyau serait le Bureau, qui ne coûterait rien à l'État. Le ministre de la Sécurité publique, M. Jacques Dupuis (successeur de M. Chagnon), exprime cette posture libérale classique comme suit: «On veut que l'industrie s'autorégularise elle-même et existe à partir des contributions de ses membres.» (Consultation 3, Dupuis).

L'autorégulation passe par le Bureau, constitué en majorité par l'industrie. C'est pourquoi on lui attribue tant de pouvoirs réglementaires, et même si le ministre se réserve le droit d'intervenir à tout moment, on comprend qu'il n'est pas dans ses intentions d'utiliser ce droit trop souvent. Au contraire, ce désengagement visible inquiète plusieurs franges des acteurs impliqués, notamment les syndicats et la police, qui plaident pour la mise en place d'un code de déontologie pour limiter l'indépendance et la liberté de l'industrie. Du fait que le Bureau soit responsable en même temps de l'implantation de normes de comportement et de leur respect, que les pouvoirs législatifs et exécutifs soient ainsi réunis en une seule instance, certains acteurs se questionnent sur les abus que cette centralisation des pouvoirs pourrait apporter. D'où la volonté d'implanter un code de déontologie, dont le respect serait assuré par des instances indépendantes, de manière à protéger le public, idée défendue par des membres de l'opposition et par la police:

Lautorégulation de l'industrie n'est pas mauvaise en soi, mais uniquement s'il est question ici de discipline entre pairs. Mais, si nous parlons de déontologie, c'est-à-dire du rapport avec le public, une instance indépendante du Bureau se doit d'être mise de l'avant, particulièrement si ce dernier est contrôlé par l'industrie via un conseil d'administration représentant majoritairement cette dernière. Les intérêts et les droits du grand public se doivent d'être protégés. (Consultation 2, FPPM) 
Si la version finale du projet n'a nullement tenu compte de ces objections, elles n'en mettent pas moins en lumière l'importance du futur Bureau, objet de toutes les convoitises et de multiples inquiétudes. Ce n'est pas un hasard si l'une des exigences majeures des intervenants était d'obtenir un siège au Bureau. Même ceux qui essayaient de se soustraire à la loi, invoquant des activités de sécurité purement internes sans lien avec le public ou la jurisprudence sur la régulation des entreprises interprovinciales, demandaient au gouvernement de leur accorder une place au Bureau. Les acteurs ont rapidement compris que celui-ci occuperait une place centrale, que ses pouvoirs seraient élargis et qu'il n'était nullement dans l'intention du gouvernement de limiter son autonomie. Or, la nomination des 11 membres du Bureau doit suivre une logique précise, dépendant largement du gouvernement. Tout d'abord, celui-ci nommera quatre de ses membres. Ensuite, si les sept restants seront nommés par «les associations représentatives de la sécurité privée» (Québec, 2006a, chapitre III.II. art. 44, §2), il revient au ministre de déterminer quelles associations sont représentatives ou pas. De ce fait, les débats se sont centrés en large partie autour des plaidoyers des différents acteurs pour être inclus dans la constitution du Bureau.

Le principal défi relatif à cette tâche consiste à représenter l'ensemble des intérêts de l'industrie en seulement sept nominations. Cette restriction a pour conséquence l'émergence d'une multitude d'associations «représentatives» qui chacune veut sa place au Bureau. L'argument pouvait ainsi varier entre une représentativité «quantitative» et «qualitative», dépendamment de la taille des associations: un grand groupe misant sur le nombre d'employés - ou sur le chiffre d'affaire - qu'il représentait; un plus petit regroupement vantant la réalité particulière de son environnement, spécificité qui demanderait une pleine représentation au Bureau. Prenons ainsi l'exemple de la dichotomie entre petites et grosses entreprises. L'une des inquiétudes des petits et moyens entrepreneurs concerne l'augmentation des frais d'exploitation liés, d'une part, à l'augmentation des exigences de formation et, d'autre part, à la multiplication des permis (un permis est nécessaire pour chacun des secteurs d'activités; Ibid, art. 4). Or, il est évident que ces frais, notamment ceux liés au second point, seront bien plus aisément amortis par une grande organisation que pour une petite entreprise qui vient de démarrer. D'autant plus que, malgré la division sectorielle du champ de la sécurité telle qu'elle est exprimée par la loi, la réalité se dessine tout autrement sur le terrain où il n'est pas rare de mêler plusieurs types 
d'activités dépendamment des opportunités du marché. Ainsi, un ancien policier qui lancerait sa propre entreprise de gardiennage ne refusera pas un travail d'investigation qu'un client lui proposerait. Si ce n'est qu'avec l'obligation de posséder un permis pour chacun des secteurs d'activités, ce nouvel entrepreneur ne pourra vraisemblablement honorer ce genre de proposition et se verra par là même pénalisé par rapport à d'autres concurrents plus imposants. Ainsi, par cet article en apparence anodin, le gouvernement a peut-être transformé le paysage de l'industrie de la sécurité en contraignant les petits joueurs à se cantonner à l'exercice d'un seul type d'activité. Cet exemple nous montre bien comment les futures décisions du Bureau de la sécurité privée pourront être d'un impact certain sur le champ de la sécurité et pourquoi sa constitution sera d'une importance cruciale.

Ce problème de représentation n'a pas été encore résolu, la loi n'ayant pas encore pris effet. Néanmoins, on comprend déjà que le Bureau sera l'un des futurs lieux de pouvoir de la sécurité privée au Québec. En visant l'autorégulation, le gouvernement n'a d'autre choix que de donner suffisamment de pouvoir à la future organisation pour permettre son autonomie. On pourrait donc dire que l'État vient de créer ici un "nœud de gouvernance», (Shearing et Wood, 2000), une source hybride et autonome de distribution et de contrôle de la sécurité. Le Bureau va délivrer les permis, décider de normes de comportement et pénaliser si besoin est ceux qui contreviennent à ces normes, ce qui fait de lui une source non négligeable de production et de contrôle de la sécurité. Toutefois, si cette autonomisation, ou autorégulation, est indéniable, une complète indépendance vis-à-vis de l'État n'est pas pour autant vraie, et ce, sur au moins deux points. En premier lieu, parce que le Bureau, bien que majoritairement aux mains de l'industrie (sept postes sur 11), est néanmoins régulé, c'est-à-dire dirigé par l'État. En ce sens, toute législation est un acte de "steering» (Rose et Miller, 1992), aussi minimale que soit l'intervention. En d'autres termes, il est possible de s'attarder sur le fait que cette nouvelle loi va accorder à l'industrie des pouvoirs certains, mais il ne faut pas perdre de vue que la décision de réguler l'industrie a été prise par le gouvernement, qui aurait pu laisser les choses en place et se contenter de la loi de 1962. On se trouve ici dans une situation où la Loi sur la sécurité privée est autant une réaffirmation de l'État dans le contrôle et la production de la sécurité qu'une acceptation que ce bien collectif soit aussi l'affaire d'intérêts privés. 
Ensuite, parce qu'il ne faut pas sous-estimer l'apport en capital symbolique que représente cette nouvelle législation pour l'industrie de la sécurité privée. Tous les intervenants impliqués dans le secteur privé ont accueilli à bras ouverts la modernisation de la Loi sur la sécurité privée. Comme le dit M. Jacques Boucher, de l'AQIS: «On applaudit ça, le fait qu'on va légiférer et qu'on va imposer une formation. On va, par là même, professionnaliser notre métier [...], on va nous reconnaître une fois pour toutes et entourer ça avec une réglementation qui va être adéquate» (Consultation 3, AQIS).

En ce sens, et comme nous l'a montré une série d'entrevues avec des gestionnaires de la sécurité privée conduites dans une recherche précédente (Mulone et Dupont, à paraître), l'État possède une capacité de légitimation qui fait cruellement défaut à l'industrie. Devenir, pour reprendre les mots de l'ancien ministre de la Sécurité publique, la «quatrième patte» (Consultation 1, Chagnon) de la sécurité (avec la police, le renseignement et l'armée) constitue une reconnaissance non négligeable pour l'industrie.

Pour poursuivre la réflexion sur les rapports de l'industrie de la sécurité privée avec le secteur public et si, comme le souhaite le ministre, l'autorégulation est atteinte par la constitution du Bureau, il nous faut maintenant nous questionner sur ce qu'il en est de l'idée de partenariat entre public et privé, entre police et sécurité privée, idée soulevée au tout début du processus législatif, lors de la rédaction du Livre Blanc.

\section{Obligation de dénonciation, rapport des sphères publique} et privée et statut de l'agent de sécurité

Le projet de loi prévoyait d'instaurer une obligation de dénonciation à la police pour trois types de crimes: contre l'ordre public (Québec, 2006a, art. 90.1), dans le cas d'une infraction relative au terrorisme (Ibid, art. 90.2) et d'une infraction mettant en danger l'intégrité physique d'une personne (Ibid, art 90.3). Ce lien officiel entre les forces de police et la sécurité privée que la loi cherchait à instituer a été fort controversé et remis en question, autant par les forces de police (qui exigeaient que la dénonciation ne s'applique pas seulement à certains crimes, mais englobe l'ensemble du Code Criminel) que par l'industrie, pour qui la délation ne peut s'appliquer aux agents, ceux-ci devant être considérés comme de simples citoyens. 
Deux questions fondamentales découlent de cette obligation de dénonciation. En premier lieu, elle institue des liens officiels entre la police et la sécurité privée, entre les sphères publique et privée, qui nous renseignent, d'une part, sur la gouvernance de la sécurité et, d'autre part et de manière plus large encore, sur les rapports des différents acteurs du champ de la sécurité. Ensuite, elle touche au statut de l'agent de sécurité privée, en ce que l'on considère ce dernier comme un simple citoyen ou comme un agent spécial - non pas un agent de la paix, mais quelque chose qui s'en rapprocherait - dont les obligations légales différeraient de celles du citoyen. La citation suivante montre comment la question de la dénonciation est intimement imbriquée dans celle du statut de l'agent de sécurité:

Moi, ce que je mettrais comme obligation de dénonciation dans le projet de loi, ce qui devrait être clarifié à mon avis, c'est l'obligation de dénoncer les crimes contre la personne. Autrement dit, il y aurait, dans le projet de loi, une obligation faite aux agents de sécurité privée ou aux agences de sécurité privée de dénoncer tout crime ou toute information qui pourrait leur laisser croire raisonnablement qu'un crime contre l'État va être commis ou qu'un crime contre la personne va être commis. Il devrait y avoir une obligation de dénonciation. Je vous le dis tout de suite, $\mathrm{M}^{\mathrm{e}}$ Castiglio [conseiller juridique de l'APPQ], que certains me disent: «Pas sûr que ce soit constitutionnel, ton affaire.» Mais j'ai demandé de regarder ça pour qu'on soit capable. Parce qu'évidemment, là, on joue dans le statut d'agent de la paix puis on ne veut pas donner un statut d'agent de la paix aux agents de sécurité privée. (Consultation 4, Dupuis)

On sait que l'ensemble du chapitre qui instaurait cette obligation de dénonciation a été retiré de la version finale de la loi. Malgré tout, la tentative d'établissement de liens officiels entre les sphères publique et privée, tout comme son échec, sont des éléments de réflexion intéressants.

Tout d'abord, parce qu'il n'existe que peu ou pas, à notre connaissance, de liens officiels entre la police et la sécurité privée ${ }^{17}$. L'exemple du centre de Philadelphie et de son BID $^{18}$ où la police, des agents de

17. Il est entendu, toutefois, que si les partenariats officiels sont rares, les contacts officieux, eux, se multiplient. Il existe ainsi un volume important d'échanges d'informations - quelquefois aux limites de la légalité - entre police publique et sécurité privée, notamment dans le secteur de l'enquête privée, un secteur fréquemment occupé par d'anciens policiers (Boone, 1993; Shapland et Van Outrive, 2000; Commission du droit du Canada, 2006).

18. Business Improvement District. 
sécurité et des propriétaires immobiliers participent ensemble à la sécurité du quartier, est lui-même plutôt exceptionnel et géographiquement limité (Greene et al., 1995; Vindevogel, 2004; Huey et al., 2005). Or, ce que proposait la loi était d'autant plus étonnant qu'en lieu et place d'un véritable partenariat, c'était une obligation légale qui était imposée, obligation qui allait de la sécurité privée vers la police et non l'inverse. En d'autres termes, on cherchait à instituer un rapport de domination de la police sur les agents de sécurité, un lien qui aurait clairement mis une partie de l'industrie de la sécurité dans une position inférieure, avec des devoirs à accomplir. On saisit rapidement que cela aurait été très contraignant, d'une part parce que coûteux en temps et en argent, d'autre part parce que liant la sphère privée au corps public, limitant par là même sa liberté. Plus symboliquement, cette contrainte légale indiquait que certains législateurs considéraient qu'une allocation de pouvoirs supplémentaires à la sécurité privée ne pouvait aller sans ajout de responsabilités en contrepartie. Trois remarques peuvent être faites à l'abandon de cette obligation.

Premièrement, le fait que la sécurité privée ait eu gain de cause montre - de même que l'ensemble du processus de négociation qui a abouti à la Loi sur la sécurité privée - combien l'industrie est aujourd'hui capable de faire du lobbying efficace, d'autant plus sur un point qui fut âprement combattu par les syndicats policiers.

Ensuite, cela nous éclaire sur la stratégie générale de conquête du champ par l'industrie de la sécurité privée. Alors que l'on s'inquiète souvent, et à raison, de la transformation de certaines entreprises de gardiennage en sorte de "parapolice» (Rigakos, 2001), nos données nous indiquent que l'objectif principal de l'industrie est avant tout de conserver un maximum de liberté, de limiter au mieux les contraintes externes qui nuiraient au libre échange et au marché de la sécurité, même si cela doit signifier des pouvoirs limités. En ce sens, l'industrie de la sécurité privée ne veut en aucun cas remplacer la police, ne voulant pas s'encombrer de la masse de contraintes qui pèse sur les organisations policières (certains, à l'instar de Crawford (2006), avancent même l'hypothèse que ce serait plutôt la police qui chercherait, par exemple par la création d'officiers au rabais, à concurrencer l'industrie sur son propre terrain). Lacceptation immédiate et sans condition par l'industrie d'une identification extérieure suffisamment éloignée de celle de la police pour éviter toute confusion du public (à la requête des syndicats policiers) montre bien que la sécurité cherche à se tailler une place à 
part dans le champ et non pas, pour le moment du moins, à remplacer des acteurs déjà existants.

Enfin, et faisant suite à cette seconde remarque, il faut souligner la possibilité que possède l'État d'imposer des obligations légales qui limiteraient le pouvoir d'action de l'industrie. Ainsi, il existe une sorte de menace potentielle qui planerait au-dessus des activités de sécurité privée. Bien sûr, nos données montrent, comme nous le disions cidessus, que l'industrie est capable de procéder à un lobbying efficace. Il est toutefois permis de penser que si les exigences de l'industrie ont si bien été écoutées, c'est parce qu'elles trouvaient un écho parfait dans les désirs de l'État. L'obligation de dénonciation n'aurait été qu'un résidu du Livre Blanc, gardé pour rassurer le public et la police peut-être, mais finalement vu comme non nécessaire et contreproductif. On peut néanmoins émettre l'hypothèse que si des pouvoirs supplémentaires (amendes, arrestations) devaient être alloués à l'industrie, cela pourrait s'accompagner de contraintes en parallèle. De récents développements ${ }^{19}$ nous indiquent que cela n'est pas forcément le cas, mais cela ne signifie pas pour autant qu'une réappropriation par l'État d'une partie de l'industrie de la sécurité privée soit inimaginable. En d'autres termes, il existe une possibilité que l'accroissement de pouvoir de l'industrie privée dans le champ de la sécurité ait comme réponse une intégration par le secteur public.

Dès lors, l'État se distingue à nouveau de tous les autres nœuds de gouvernance par cette capacité de lier l'industrie par des obligations légales et de renforcer son contrôle. Bien sûr, cela n'irait pas sans coûts supplémentaires, coûts que l'État ne serait peut-être pas prêt à assumer. Nous ne disons pas non plus que la position particulière qu'occupe l'État dans la gouvernance de la sécurité soit immuable. Elle possède néanmoins actuellement des caractéristiques qui le différencient radicalement des autres nœuds de gouvernance, les différentes secteurs de l'industrie privée en tête.

19. Notamment, le pouvoir d'utiliser la force alloué par un contractant privé à une agence de gardiennage, délégation qui se passe de l'État et se déroule en circuit fermé. Voir l'article du Washington Post daté du 2 janvier 2007, p. A04, disponible à l'adresse suivante: http://www.washingtonpost.com/wp-dyn/content/article/2007/01/01/AR 2007010100665.html. 


\section{Conclusion}

On peut voir la Loi sur la sécurité privée comme une réappropriation du territoire de la sécurité par l'État, dans la mesure où tout processus de régulation l'est. S'il est vrai que cette loi est libérale et donne en ce sens une assise à l'industrie, il n'en reste pas moins que l'État, en légiférant, réaffirme son statut proéminent sur la délivrance du bien collectif «sécurité». À partir de cette constatation générale, un certain nombre d'observations ont pu être mises en avant.

1. L'industrie est devenue suffisamment importante pour faire pencher la balance gouvernementale en sa faveur, même si cela est peut-être dû à une compréhension mutuelle qui a précédé le processus législatif, notamment à la suite d'une implication importante de l'industrie, et ce, dès les premières étapes qui ont amené à l'adoption du projet de loi 88. Il serait intéressant de voir ce qui se passerait dans le cas d'une loi dont le contenu serait profondément en conflit avec les intérêts de l'industrie.

2. Le Bureau est un nœud de pouvoir et de gouvernance, et sa constitution représente une modification significative du paysage de la gouvernance de la sécurité. Toutefois, l'État continue à occuper une place particulière dans la gouvernance de la sécurité, et ce, sur deux points: il légifère certes, mais, en sus, il légitime une industrie en lui donnant une «reconnaissance». Cette capacité de conférer du capital symbolique s'accompagne d'une menace potentielle, mais constante, de contrôle et de réappropriation.

3. L'industrie n'est pas un bloc monolithique et homogène. Elle est traversée par des intérêts divergents dont il faut tenir compte quant à son évolution (grandes entreprises versus PME, distinctions sectorielles, entreprises nationales versus locales...). Dès lors, il est quelque peu abusif de parler d'une relation entre l'État et la sécurité privée, tout comme il faudrait parler des sécurités privées. Bien que nous ayons mis de l'avant quelques exemples de relations différentielles entre certaines parties de l'industrie et l'État, il conviendrait, dans de futures recherches, d'investiguer plus loin ce rapport différencié et d'aller voir quels secteurs de l'industrie sont plus à même d'être dépendants des agences de régulation étatiques ou de la police et comment ces différences agissent sur la gouvernance de la sécurité.

4. La délimitation du secteur de la sécurité constitue l'une des futures matières à débat. Avec la constitution du Bureau, et le développement de nouvelles législations un peu partout à travers le monde (les provinces 
de l'Alberta et de l'Ontario sont en train de travailler à de nouvelles législations), pouvoir déterminer ce qui est de la sécurité et ce qui n'en est pas devient primordial. Sur ce point, il conviendrait de continuer à faire des recherches pour mieux cerner et définir ce que l'on entend par sécurité privée. Bien que les avancées faites par la Loi sur la sécurité privée (relativement à l'ancienne législation de 1962) soient intéressantes, elles demeurent insuffisantes au vu du manque de précision de chacune des activités sectorielles. Ce flou pourra ainsi être instrumentalisé par le Bureau, notamment par l'attribution de permis d'agent et d'agence, de façon à favoriser une partie de l'industrie par rapport à une autre.

5. La sécurité privée ne veut pas remplacer la police, du moins pour le moment. Les discours de type «parapolice» sont souvent le fait des individus sur le terrain (les gardes de sécurité) (Rigakos, 2001 ; Manzo, 2006) et ne se retrouvent nullement dans les propos des gestionnaires. Si l'on peut interpréter cela comme une tentative de séduction et un évitement de confrontation directe, il ne faut pas abandonner l'hypothèse d'une réelle logique, soutenue par une volonté de liberté d'action et d'occupation d'une place spécifique dans le champ, d'une place unique et irremplaçable. Cela ne signifie pas que la sécurité privée ne va pas chercher à occuper, à long terme, des lieux d'activités traditionnellement réservés au secteur public. Toutefois, sa stratégie présente consiste plutôt à construire et à consolider une niche dans le champ de la sécurité de laquelle elle ne pourra pas facilement être délogée.

6. En dehors d'une place permanente au Bureau, la police n'a rien obtenu, sans compter que seuls les syndicats policiers se sont sentis véritablement concernés par la nouvelle loi. Si on peut conclure, dans un premier temps, que la police est «hors champ», cela pourrait plutôt dénoter de la transformation (commodification) et de la commercialisation rampante de l'objet sécurité (Loader, 1999; Gans, 2000; Crawford et al., 2003). En d'autres termes, de bien collectif la sécurité se transforme en bien marchand et la police veut obtenir sa part du gâteau. La question n'est plus de savoir si l'on doit contrôler le développement de la sécurité privée, ou même si l'on doit mieux circonscrire les responsabilités de chacun, mais plutôt de savoir qui va le mieux s'en tirer dans la course aux profits que le marché de la sécurité génère. Si certains auteurs voient dans cette compétition une défaite irrémédiable de la police (Jones et Newburn, 1998), de futures recherches devront nous permettre de mieux statuer sur les chances de chacun. Ce qui est sûr, 
c'est que cette transformation risque d'accentuer le flou de la séparation entre secteur privé et public.

$\mathrm{Au}$ vu de nos analyses, il convient, pour conclure, de se questionner sur l'applicabilité du modèle de la gouvernance nodale à la réalité québécoise. Alors qu'on observe effectivement une prolifération d'acteurs privés et hybrides, ainsi qu'une complexification du champ rendant la perspective wébérienne de l'État centralisateur de plus en plus éloignée de la réalité, il n'en reste pas moins que nos données ont pu mettre en avant certaines caractéristiques propres à ce dernier, soit ses capacités à légitimer et à légiférer.

Dès lors, on peut faire l'hypothèse que la gouvernance nodale ne peut fonctionner qu'en intégrant la capacité légitimante de l'État dans la production de sécurité (Sarre et Prenzler, 2005). Il est par ailleurs intéressant de souligner que cette même gouvernance nodale a été en partie élaborée à partir d'expériences pilotes concluantes conduites en Afrique du Sud (Shearing et Wood, 2003), pays dont le gouvernement ne possède peut-être pas autant de capacité de légitimation vis-à-vis du public que la plupart des pays occidentaux. Sans parler de réfutation de la gouvernance nodale, il nous semble important de poser certaines limites quant à son application. Alors qu'elle semble toute indiquée dans des pays où l'État connaît un déficit de légitimité, elle risque de se confronter à de nombreuses difficultés au sein de pays dont l'État est fort, à moins qu'elle ne s'applique dans des zones où cette même légitimité est défaillante, telles que les ghettos que l'on peut trouver dans certaines villes états-uniennes ou dans certaines banlieues françaises. Au Canada, l'État continue de tenir une place centrale et unique dans la gouvernance de la sécurité, ce qui ne doit pas pour autant nous faire oublier que la sécurité privée se met elle aussi à y jouer un rôle de plus en plus important, ce que la Loi sur la sécurité privée ne fera qu'exacerber.

\section{Références}

Bayley, D. H., \& Shearing, C. (2001). The new structure of policing. Washington, D. C.: The National Institute of Justice, U. S. Department of Justice.

Boone, K. (1993). La fonction d'enquête dans le secteur privé: Développements et conséquences pour les polices publiques. Déviance et Société, 17 (2) 184 208.

Brink's Canada ltée. (2005). Mémoire présenté à l'Assemblée nationale du Québec. Commission des institutions. Projet de loi $n^{\circ} 88$, Loi sur la sécurité privée. Ministère de la Sécurité publique. Québec: Bibliothèque nationale du Québec. 
Brodeur, J.-P. (2003). Les visages de la police: Pratiques et perceptions. Montréal: Presses Universitaires de Montréal.

Burbidge, S. (2005). The governance deficit: Reflections of the future of public and private policing in Canada. Canadian Journal of Criminology and Criminal Justice, 47 (1), 63-86.

Commission du droit du Canada (2006). En quête de sécurité: L'avenir du maintien de l'ordre au Canada. Ottawa: Ministère des Travaux publics et des Services gouvernementaux.

Crawford, A. (2006). Policing and security as 'club goods' : the new enclosures? In J. Wood \& B. Dupont (eds.), Democracy, society and the governance of security (111-138). Cambridge: Cambridge University Press.

Crawford, A., Lister, S., \& Wall, D. (2003). Great expectations: Contracted community policing in New Earswick. York: Joseph Rowntree Foundation.

Dupont, B. (2005). Les morphologies de la sécurité après le 11 septembre: hiérarchies, marché et réseaux. Criminologie, 38 (2), 123-155.

Dupont, B. (2006a). Mapping security networks: From metaphorical concept to empirical model. In J. Flemming \& J. Wood (Eds.), Fighting crime together: The challenges of policing and security networks (35-59). Sydney: University of New South Walles Press.

Dupont, B. (2006b). Delivering security through networks: Surveying the relational landscape of security managers in an urban setting. Crime, Law and Social Change, 45, 165-184.

Gans, J. (2000). Privately paid public policing: law and practice. Policing e Society, 10 (2), 183-206.

Garland, D. (2001). The culture of control: Crime and social disorder in contemporary society. Chicago: Chicago Press University.

Greene, J. R., Seamon, T. M., \& Levy, P. R. (1995). Merging public and private security for collective benefit: Philadelphia's center district. American Journal of Police, 14 (2), 3-20.

Huey, L. J., Ericson, R. V., \& Haggerty, K. D. (2005). Policing fantasy city. In D. Cooley (Ed.), Re-imagining policing in Canada (140-208). Toronto: University of Toronto Press.

Johnston, L. (1992). The rebirth of private policing. New York: Routledge.

Johnston, L. (2000). Policing Britain: Risk society and governance. Harlow: Longman.

Johnston, L., \& Shearing, C. (2003). Governing security: Explorations in policing and justice. London: Routledge.

Jones, T., \& Newburn, T. (1998). Private security and public policing. Oxford: Clarendon Press.

Jones, T., \& Newburn, T. (Eds.) (2006). Plural policing: A comparative perspective. New York: Routledge.

Loader, I. (1999). Consumer culture and commodification of policing and security. Sociology, 33 (2), 373-392. 
Loader, I., \& Walker, N. (2001). Policing as a public good: Reconstituting the connections between policing and the state. Theoretical Criminology, 5, 935.

Loader, I., \& Walker, N. (2006). Necessary virtues: The legitimate place of the state in the production of security. In J. Wood \& B. Dupont (Eds.), Democracy, society and the governance of security (165-195). Cambridge: Cambridge University Press.

Manzo, J. (2006). You can't rent a cop: Mall security officers' management of a "stigmatized" occupation. Security Journal, 19 (3), 196-210.

Mulone, M., \& Dupont, B. (à paraître). Gouvernance de la sécurité et capital: Les gestionnaires de la sécurité privée. Déviance et Société.

Rigakos, G. S. (2001). The new parapolice: Risk markets and commodified social control. Toronto: University of Toronto Press.

Rose, N., \& Miller, P. (1992). Political power beyond in the State: Problematics of government. The British Journal of Sociology, 43 (2), 173-205.

Sanders, T. (2005). Rise of the Rent-a-Cop: Private security in Canada, 19912001. Canadian Journal of Criminology and Criminal Justice, 47 (1), 175-190.

Sarre, R., \& Prenzler, T. (2005). The law of private security in Australia. Pyrmont: Thomson Lawbook Co.

Shapland, J., \& Van Outrive, L. (Eds.) (2000). Police et sécurité: Contrôle social et interaction public/privé. Paris: L'Harmattan.

Shearing, C. (2005). Nodal security. Police Quarterly, 8 (1), 57-63.

Shearing, C. (2006). Reflections on the refusal to acknowledge private governments. In J. Wood \& B. Dupont (Eds.), Democracy, society and the governance of security (11-32). Cambridge: Cambridge University Press.

Shearing, C., \& Wood, J. (2000). Reflections on the governance of security, a normative inquiry. Police Practice, 1 (4), 457-476.

Shearing, C., \& Wood, J. (2003). Governing security for common goods. International Journal of the Sociology of Law, 31, 205-225.

Vindevogel, F. (2004). Les municipalités favorisent-elles l'émergence de policiers privés? Déviance et Société, 28 (4), 507-532.

Wood, J., \& Shearing, C. (2007). Imagining security. Portland: Willan Publishing.

\section{Lois et jurisprudence}

Québec (1962). Loi sur les agences d'investigation ou de sécurité privée. (L. R. Q., c. A-8). Règlement d'application de la Loi sur les agences d'investigation ou de sécurité privée (A-8, r. 1). Québec: Ministère de la Sécurité publique.

Québec (1989). Bell Canada c. Québec. Québec: Commission de la santé et sécurité au travail.

Québec (2003). Livre blanc: La sécurité privée, partenaire de la sécurité intérieure. Québec: Ministère de la Sécurité publique. Consulté le 12 septembre 2007 : http://www.msp.gouv.qc.ca/police/publicat/livreblanc/livre_blanc_ secsecprive_12-03.pdf 
Québec (2006a) Loi sur la sécurité privée. (L. R. Q. c. S-3.5). Québec: Ministère de la Sécurité publique.

Québec (2006b). La réforme de la loi. L'adoption de la Loi sur la sécurité privée: le couronnement de quelques années de réflexions. Québec: Ministère de la Sécurité publique. Consulté le 12 septembre 2007: http://www.msp.gouv.qc.ca/ police/ppolice.asp?txtSection $=/$ secprive $\&$ txtCategorie $=$ reforme_loi

\section{ANNEXE}

\section{Liste des participants et des abréviations}

${ }^{\star}$ : a participé aux consultations particulières.

- $\mathrm{ABC}^{\star}$ : Association des banquiers canadiens.

- ACAIQ: Association des courtiers et agents immobiliers du Québec.

- $\mathrm{APAS}^{\star}$ : Association provinciale des agences de sécurité.

- $\mathrm{APEPQ}^{\star}$ : Association professionnelle des enquêteurs privés du Québec.

- $\mathrm{APPQ}^{\star}$ : Association des policières et policiers provinciaux du Québec.

- APSPQ $^{\star}$ : Association provinciale de la sécurité privée du Québec.

- $\mathrm{AQIS}^{\star}$ : Association québécoise de l'industrie de la sécurité.

- ASIS $^{\star}$ : American society for industrial security.

- BAC $^{\star}$ : Bureau d'assurance du Canada.

- Brink's Canada ltée`.

- CANASA-Québec ${ }^{\star}$ : Association canadienne de la sécurité, section Québec.

- Chambre de la sécurité financière.

- CMEQ ${ }^{\star}$ : Corporation des maîtres électriciens du Québec.

- Conseil du patronat du Québec.

- Conseil interprofessionnel du Québec.

- CQCD: Conseil québécois du commerce de détail.

- CRARR: Centre de recherche-action sur les relations raciales.

- $\mathrm{CSN}^{\star}$ : Confédération des syndicats nationaux.

- $\mathrm{FCEI}^{\star}$ : Fédération canadienne de l'entreprise indépendante.

- FPPM $^{\star}$ : Fraternité des policiers et policières de Montréal Inc.

- $\mathrm{FQPM}^{\star}$ : Fédération des policiers et policières municipaux du Québec.

- Garda Inc.*.

- Institut Grasset*.

- Le Protecteur du citoyen.

- Maîtres serruriers du Québec ${ }^{\star}$.

- REASINS ${ }^{\star}$ : Regroupement d'employé-e-s d'agences de sécurité et d'investigation non syndiquées.

- RESPQ ${ }^{\star}$ : Regroupement des entreprises de sécurité privée du Québec. 
- SCSGQ: Syndicat des Constables Spéciaux du Gouvernement du Québec.

- SCSI*: Société canadienne de la sûreté industrielle inc.

- $\mathrm{SNCF}^{\star}$ : Syndicat national des convoyeur-e-s de fonds.

- UASQ ${ }^{\star}$ : Union des Agents de Sécurité du Québec, Métallurgistes Unis d'Amérique, Section locale 8922 FTQ

- Université de Montréal. 